\title{
RENOPROTECTIVE ROLE OF TUALANG HONEY AGAINST HIGH CHOLESTEROL DIET INDUCED ACUTE KIDNEY DISEASES IN AN ANIMAL MODEL
}

Zenab B. Hamad Mohamed ${ }^{1}$, Hamad Abdulsalam Hamad Alfarisi ${ }^{1}$, Nor Zamzila Abdullah $^{1}$, Naznin Muhammad ${ }^{1}$ and Roslina Abdul Rahim ${ }^{1}$

${ }^{1}$ Kulliyyah of Medicine, International Islamic University Malaysia

Presenter: Zenab B. Hamad Mohamed, whiitefllower@gmail.com

Introduction: Although there is a growing insight into the causes and mechanisms of kidney diseases, preventive and therapeutic measures are still few. The aim of this study was therefore to determine the renoprotective effect of tualang honey against high cholesterol diet induced acute kidney disease in an animal model.

Materials and method: Ten female Sprague-Dawley rats were divided into two groups: high cholesterol diet (HCD) group, fed with $12 \%$ cholesterol diet with $0.3 \%$ cholic acid, and high cholesterol diet with tualang honey $(H C D+T H)$ group, fed with HCD with oral daily dose of $1.4 \mathrm{~g} / \mathrm{kg} /$ day of tualang honey by gavage. Biochemical analysis of lipid profile and renal function were performed at 48 hours, 7 days, and 6 weeks of the experiment. The animals were sacrificed at 6 weeks and the kidneys were harvested for histological examination.

Results: There was no significant difference between the HCD and the HCD+TH groups in the level of TC. The mean serum LDL-C, TG and VLDL levels at completed 48 hours and 7 days for the HCD+TH group were lower as compared to the HCD group. The only significant difference in renal function test between HCD and HCD + TH groups reported were theserum creatinine at completed 48 hours. Histological examination of the kidney tissue of the HCD and HCD+TH groups at 6 weeks revealed segmental mesangial hypercellularity and mesangial matrix expansion of the glomeruli.

Conclusion: Tualang honey supplementation has resulted in an improvement of the renal profile which is suggestive of its renoprotective effect. However, the histopathological examination of the kidneys revealed similar changesin both groups and this may be related to the dose of honey used in the study. Additionally, tualang honey showed improvement in LDL-C, triglyceride and VLDL levels indicating its lipid lowering activities. 